\title{
Light and electron microscopic demonstration of extracellular immunoglobulin deposition in renal tissue
}

\author{
M D AL-NAWAB, D R DAVIES Department of Histopathology, UMDS at St Thomas's Hospital, \\ London
}

SUMMARY Extracellular immunoglobulin (IgG) deposits were shown by both light and electron microscopy in renal biopsy material using immunogold labelling. After fixation of tissue in $4 \%$ paraformaldehyde and embedding in Lowicryl $\mathrm{K} 4 \mathrm{M}$, semithin sections were cut and stained using the immunogold silver stain. The sections were then viewed and areas of interest were noted; ultrathin sections were cut from the same block of tissue, then stained using immunogold. Good localisation was achieved at both optical and ultrastructural levels allowing direct correlation to be made in the same area of tissue.

The demonstration of extracellular immunoglobulin deposition in renal tissue is a very important aspect in the diagnosis of renal disease in man. At the light microscopical level antibodies labelled with fluorescein or with peroxidase are the most widely used. A previously developed peroxidase technique' has been used to demonstrate immunoglobulin deposition both at the light and ultrastructural level in human disease. ${ }^{2}$ This pre-embedding technique and its variants have some major disadvantages, the most important of which is reliably securing adequate penetration of tissue. There is also the difficulty of defining the extent of the peroxidase reaction product and the possibility of diffusion and spurious localisation. ${ }^{3}$ The recent development of hydrophilic resins for use in electron microscopy has meant that immunolabelling after embedding can be done $e^{4}$ and avoids the potentially damaging effects of the "etching" needed with most hydrophobic resins. These methods avoid the problems of inadequate penetration of tissue by large antibody and tracer molecules because most tissue barriers are disrupted during the sectioning of the tissue, allowing direct access of immunological reagents to the interior of the cells.

Another boost to post-embedding immunostaining has been the introduction of gold labelled antibodies. Colloidal gold offers many advantages as a label: the colloidal gold particle is electron dense and has a characteristic appearance, making it easily distinguishable from biological structures. ${ }^{5}$ Colloidal gold

Accepted for publication 2 May 1989 particles can be produced in virtually any size between 1-150 nm. Particles between the sizes of 5-40 nm are the most widely used: they give more efficient labelling than larger particles because they produce less steric hindrance. ${ }^{5}$ Gold particles of this size are not visible by light microscopy, but may be rendered so by the use of silver enhancement. ${ }^{6}$ These silver enhancement tech-? niques were technically demanding and the results have been unreliable. The recent development of silver enhancement kits which are insensitive to light and are less critically dependent on ionic concentration has, in our hands, facilitated the consistent production of good light microscopic results.

The method we describe here makes use of all of the technical advances mentioned and allows direct correlation between light and electron microscopy on the same block of tissue to be made. The survival of the antigens in the resin enables post-embedding immunocytochemistry to be carried out and this has many advantages over pre-embedding techniques.

\section{Material and methods}

Fresh renal tissue was obtained from routine needle biopsies. The specimens were fixed and processed into Lowicryl K4M.

Tissue was immersion-fixed in $4 \%$ paraformaldehyde for two hours and then processed into Lowicryl $\mathrm{K} 4 \mathrm{M}$ as follows. After fixation the tissue was dehydrated in a graded series of alcohol (methanol):

$150 \%$ methanol at $-20^{\circ} \mathrm{C}$ for 30 minutes;

$280 \%$ methanol at $-20^{\circ} \mathrm{C}$ for 60 minutes;

$390 \%$ methanol at $-20^{\circ} \mathrm{C}$ for 60 minutes. 
The tissue was then infiltrated with the resin as follows:

1 pure methanol/pure Lowicryl in a 1:1 ratio at $-20^{\circ} \mathrm{C}$ for 30 minutes;

2 pure methanol/pure Lowicryl in a 1:2 ratio at $-20^{\circ} \mathrm{C}$ for 60 minutes;

3 pure Lowicryl at $-20^{\circ} \mathrm{C}$ for 60 minutes;

4 pure Lowicryl at $-20^{\circ} \mathrm{C}$ overnight.

The tissue was them embedded in fresh resin and put in gelatin capsules and photopolymerised at $-35^{\circ} \mathrm{C}$ with diffuse irradiation by ultraviolet light of $\simeq 360 \mathrm{~nm}$ wavelength overnight. The capsules were further suspended in ethanol baths with their lower poles immersed in alcohol to ensure adequate dissipation of any heat build-up inside the capsules, the polymerisation process being an exothermic reaction. The blocks were then removed and further irradiated at room temperature for 24-48 hours to ensure better sectioning properties of the blocks.

\section{IMMUNOGOLD-SILVER STAIN (IGSS)}

Semithin sections (about $2 \mu \mathrm{m}$ in thickness) were cut from the polymerised blocks using glass knives on an LKB ultramicrotome. They were applied to multispot glass slides coated with $0.05 \%$ poly-1-lysine (Sigma) to ensure that the sections would not separate during staining and washing of the slides. The slides were air dried at room temperature for one hour before further treatment.

The sections were covered in phosphate buffered saline ( $\mathrm{pH} \mathrm{7.4)}$ for two minutes before applying dilute normal goat serum ( $1 / 1$ dilution) to block non-specific binding of antibodies to the tissue. This was left for 20 minutes in a wet chamber. The goat serum was then tipped off, but not washed off, and the sides of the slide wiped without touching the section.

Varying dilutions of rabbit anti-human IgG (heavy chain) were applied to find the optimal dilution that allowed the best staining of the sections. The diluent was PBS (pH 7.4) containing $1 \%$ cold fish gelatin. ${ }^{78}$ The dilutions initially used were between $1 / 10$ to $1 / 8000$. This primary incubation was carried out for one hour in a wet chamber followed by washing of the slides in PBS ( $\mathrm{pH} \mathrm{7.4),} \mathrm{twice,} \mathrm{for} \mathrm{seven} \mathrm{and} \mathrm{a} \mathrm{half}$ minutes each with agitation. This was then followed by a 60 minute incubation with the secondary antibody ( $5 \mathrm{~nm}$ gold labelled goat anti-rabbit IgG antibody) (Jansen) in the wet chamber. The slides were removed from the wet chamber and immersed in PBS in a Coplin jar for five minutes with agitation, followed by a further wash in double distilled water twice for five minutes. The slide was dried (back and sides) with a clean drying cloth. Silver stain amplication was begun using the Intense $\mathbf{M}$ silver enhancement kit (Jansen). The reaction was allowed to continue for nine minutes before it was terminated by washing the slides in double distilled water for five minutes. Finally, the sections were counterstained with toluidine blue for two minutes, dried, and mounted.

\section{IMMUNOGOLD STAINING (IGS)}

Ultra-thin sections were cut from the same blocks of tissue used for the IGSS method using a diamond knife. Gold coloured sections (about 900 A thick) were picked up on 200 mesh nickel grids. These were left in a desiccator for one hour before staining was started.

The grids were first floated on $50 \mu$ of PBS (pH 7.4) for two minutes, followed by floating the grids on diluted $1 / 1$ goat serum for 20 minutes in a wet chamber. This was followed by a two hour incubation, with the primary antibody (rabbit anti-human IgG antibody) in a $1 / 2600$ dilution. After incubation of the grids with the primary antibody the grids were washed five times for six minutes each in PBS, pH 7.4 (drop method), and then incubated with the secondary antibody (goat anti-rabbit $10 \mathrm{~nm}$ gold labelled antibody) (Biocell) for 30 minutes, followed by four further washes of five minutes in PBS, $\mathrm{pH} 7 \cdot 4$, and twice for five minutes each in double distilled water. The grids were counterstained with aqueous uranyl acetate for 10 minutes, desiccated overnight, and viewed.

\section{CONTROLS}

Negative and positive control tests were carried out on renal tissue, where absence or presence of human IgG had been shown previously by conventional immunofluorescence studies on frozen sections. For absorption tests, the antigen (purified human IgG, about $95 \%$ pure) was purchased from Sigma, and not from the company that supplied the antibody (Dako). Characterisation of the antigen was carried out by determining the molecular weight and isoelectric point

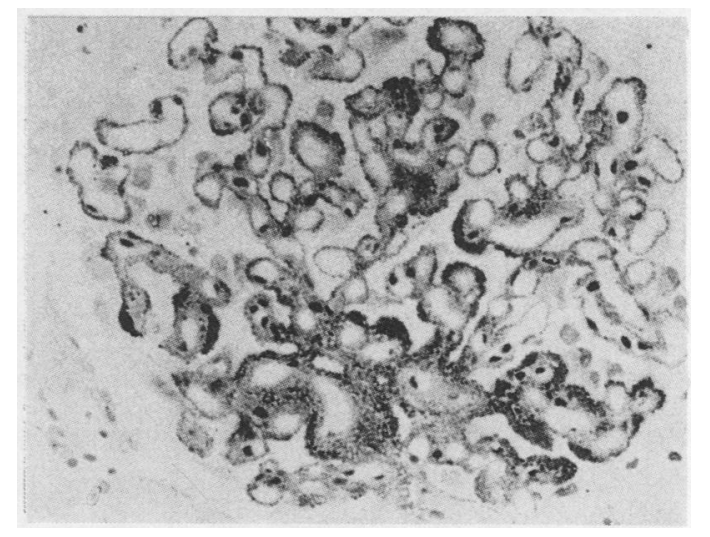

Fig 1 Lupus nephritis. Global, granular, staining in the glomerular basement membrane with heavy mesangial staining. (Indirect IGSS.) 
of the antigen. For convenience, the absorption was measured by the quantity of antigen added in $\mathrm{nmol} / \mathrm{ml}$ of optimally diluted antibody. The antigen and antibody were placed in a sealed phial and were shaken vigorously to distribute the antibody over the entire surface to make sure all the antigen was taken up. The antigen and antibody were incubated for 24 hours at $4^{\circ} \mathrm{C}$ before the solution was used for staining. As a further control, the antibody was also absorbed with inappropriate purified antigen, under the same conditions.

\section{Results}

Semithin sections from renal biopsy material embedded in Lowicryl K4M, incubated primarily in various concentrations of rabbit and anti-human IgG antibody, followed by a secondary incubation with goat anti-rabbit gold labelled antibody (GAR $10 \mathrm{~nm} \mathrm{LM}$ grade, Jansen), showed no visible staining. When the sections were further subjected to silver amplification using the Intense $M$ silver enhancement kit (Jansen), however, all concentrations of the anti-human IgG antibody except $1 / 8000$ showed a positive dark, brownish-black staining on glomerular deposits. In cases of lupus nephritis there was granular staining of

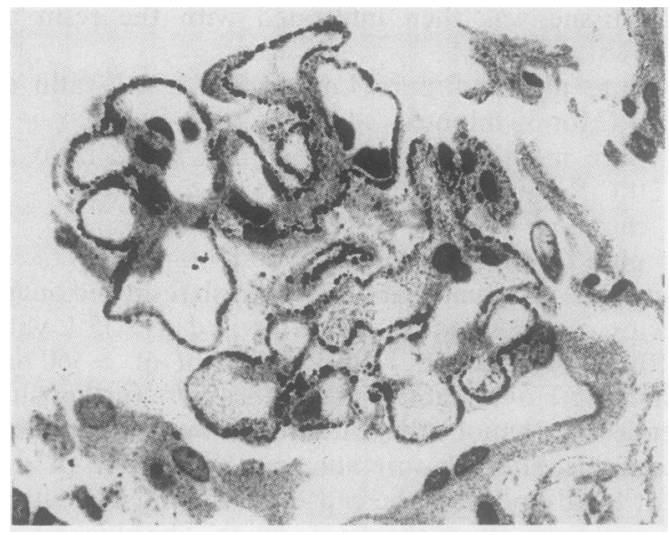

Fig 2 Idiopathic membranous glomerulonephritis. Global, granular, staining in the glomerular basement membrane without mesangial staining. (Indirect IGSS.)

the glomerular capillary walls and also of the mesangium (fig 1). Staining was also recorded in the tubular epithelial cells. In cases of idiopathic membranous glomerulonephritis staining was restricted to the glomerular capillary wall but no staining was seen in the mesangial matrix (fig 2). There was also staining of resorption granules in some tubular epithelial cells.

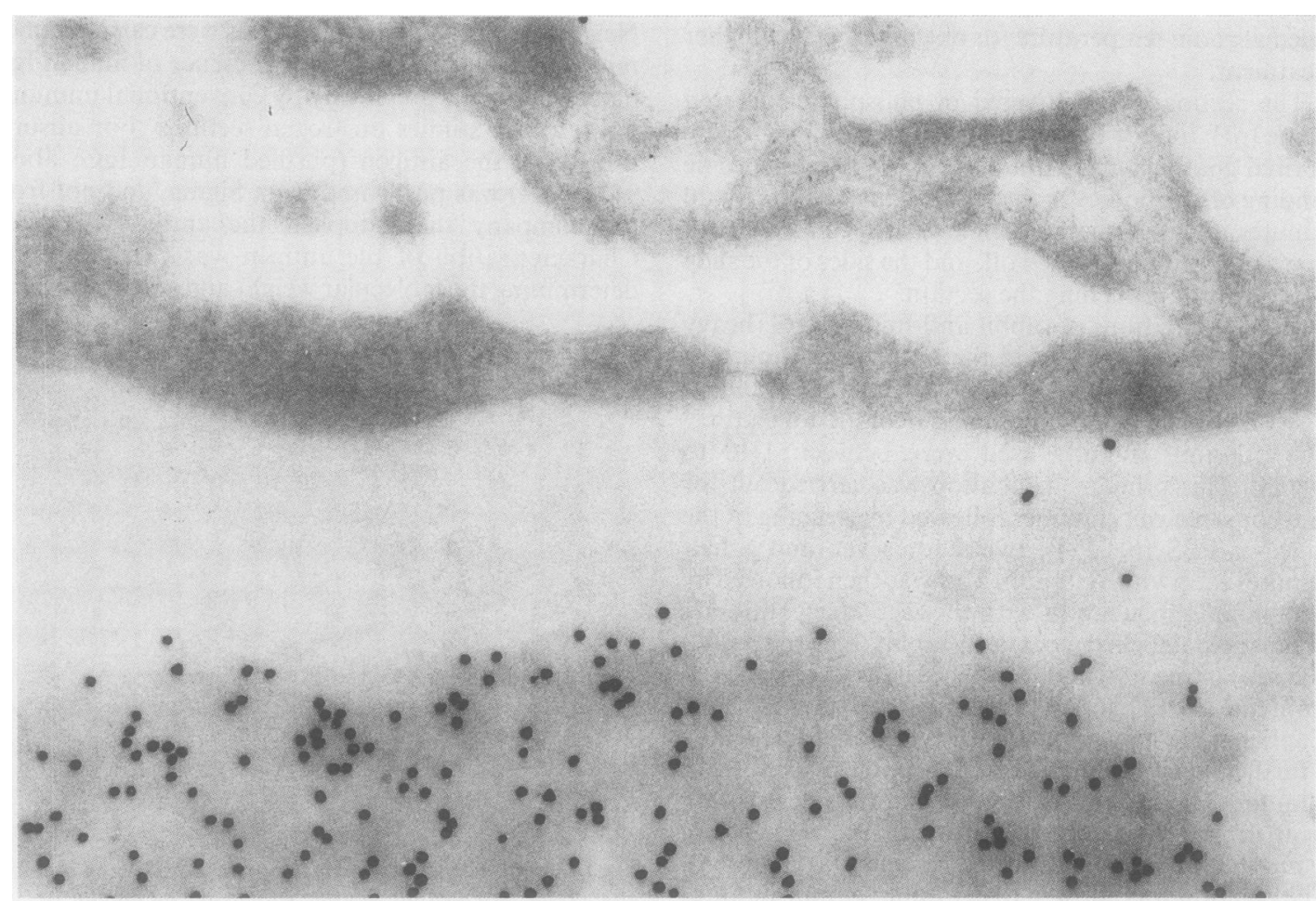

Fig 3 Electron micrograph of lupus nephritis showing labelling of a subendothelial deposit (Indirect IGS, $20 \mathrm{~nm}$ gold label.) 


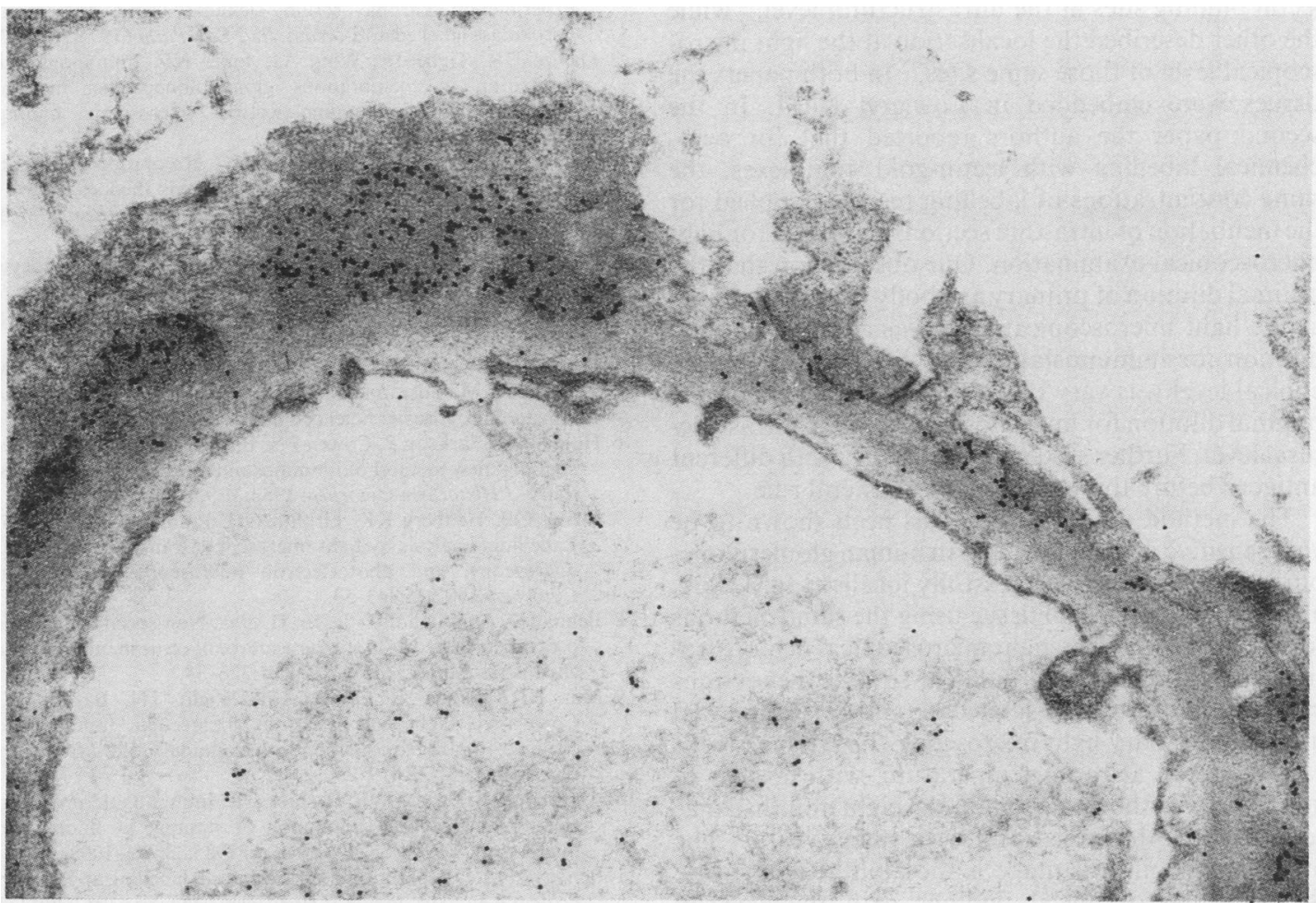

Fig 4 Electron micrograph of idiopathic membranous glomerulonephritis showing labelling of subepithelial deposits in the glomerular capillary wall. (Indirect IGS, $20 \mathrm{~nm}$ gold label.)

These staining patterns were also seen on frozen sections from the same cases using immunofluorescence. Although positive staining was observed at low dilutions, the level of background staining was unacceptable and the best signal:noise ratio was obtained at a dilution of $1 / 2600$. Both negative and positive controls confirmed the specificity and sensitivity of the method. The absorption tests showed that immunoreactivity was abolished by addition of any concentration above $0.001 \mathrm{nmol} / \mathrm{ml}$ of specific antigen.

After viewing the semithin sections, areas of interest were noted and ultra-thin sections were cut from the tissue blocks. In the cases of lupus nephritis, subepithelial, subendothelial, and mesangial electron dense deposits were intensely labelled with gold particles (fig 3 ). In the cases of idiopathic membranous glomerulonephritis subepithelial electron dense deposits were intensely labelled but no subendothelial labelling could be seen (fig 4). The best immunolabelling was obtained at a dilution of $1 / 2600$, the same dilution that was considered to be optimal for staining at the light microscopical level.

\section{Discussion}

A disadvantage found in most immunohistochemical techniques is the absence of direct correlation between light and electron microscopical images, although some techniques have been reported to achieve direct correlation between the optical and ultrastructural levels. ${ }^{2910}$ The combination of techniques we have described here allows light microscopical immunohistochemistry on one section to be compared with ultrastructural immunohistochemistry of an adjacent section from the same block. There is also the added advantage of the possibility of staining ultra-thin sections for different antigens." The methods described here avoid such problems as the impermanence of fluorescein labelled preparations, the penetration difficulties of pre-embedding techniques, and the possible lack of precision of the DAB reaction product in peroxidase ultrastructural immunohistochemistry.

Roth et al described the use of lectin-gold complexes in various animal tissues in two papers published four years apart. The first paper described localisation of 
lectin binding sites at the ultrastructural level, ${ }^{12}$ while the other described the localisation at the light microscopical level of those same sites. ${ }^{13}$ In both papers the tissues were embedded in Lowicryl K4M. In the second paper the authors reported that for cytochemical labelling with lectin-gold complexes, the same concentrations of labelling reagents applied for the incubation of ultra-thin sections were used for light microscopical examination. Our observation that the optimal dilution of primary antibody used for staining at the light microscopical level was also the optimal dilution for immunostaining at the electron microscopical level was very time saving in determining the optimal dilution for immunostaining at the ultrastructural level. Further studies are necessary with different antigens before this can be made a general rule.

The method described here has been shown to be very sensitive in localising IgG in human glomerulonephritis. We have also successfully localised IgM, IgA, and $\mathrm{C} 3$ in human renal tissue using the same methods (data not shown). The light microscopical demonstration of the antigen is permanent, so that the sections can be referred to at a later date and can be viewed using an ordinary light microscope. The tissue blocks can be stored at room temperature, with no loss of antigenicity in the tissue for at least eight months, or as reported by other researchers, for many years. ${ }^{10}$ The most important advantage is the ability to correlate the light microscopical findings with the electron microscopical level on the same block of tissue. These virtues make the method potentially valuable in histopathology.

\section{References}

1 Davies DR, Clark AE. Demonstration of immunoglobulin containing deposits in glomerular basement membrane in experimental chronic serum sickness using horse-radish peroxidase labelled anti-serum. Br J Exp Pathol 1975;56:28-33.

2 Davies DR, Tighe JR, Wing AJ, Jones NF. Immunoglobulin deposition in membranous glomerulonephritis: immunofluorescence and immuno-electron microscopy findings. Histopathology 1977;1:39-52.

3 Courtoy PJ, Picton DH, Farquhar MG. Resolution and limitations of the immunoperoxidase procedure in the localisation of extracellular matrix antigens. $J$ Histochem $C y$ tochem 1983;31: 945-51.

4 Causton BE. The choice of resins for electron immunocytochemistry. In: Polak JM, Varndell M, eds. Immunolabelling for electron microscopy. Amsterdam: Elsevier Science Publishers, 1984:29-51.

5 Romano EL, Romano M. Historical aspects. In: Polak JM, Varndell M, eds. Immunolabelling for electron microscopy. Amsterdam: Elsevier Science Publishers, 1984:3-15.

6 Holgate CS, Jackson P, Cowen PN, Bird CC. Immunogold-silver staining: new method of immunostaining with enhanced sensitivity. J Histochem Cytochem 1983;31:938-44.

7 Birrell GB, Hedberg KK, Griffith OH. Pitfalls of Immunogold Labelling: analysis by light microscopy, transmission electron microscopy, and photoelectron microscopy. J Histochem Cytochem 1987;35:843-53.

8 Behnke O, Ammitzboll T, Jessen H, et al. Non-specific binding of protein-stabilized gold sols as a source of error in immunocytochemistry. Eur J Cell Biol 1986;41:326-38.

9 Mar H, Tsukada T, Gown AM, Wight TN, Baskin DG. Correlative Light and Electron Microscopic Immunocytochemistry on the Same section with Colloidal Gold. J Histochem Cytochem 1987;35:419-25.

10 Alexander RB, Isaacs WB, Barrack ER. Immunogold probes for electron microscopy: evaluation of staining by fluorescence microscopy. J Histochem Cytochem 1985;33:995-1000.

11 Bendayan M. Double immunocytochemical labelling applying the protein A-gold technique. J Histochem Cytochem 1982;30:81-5.

12 Roth J. Application of lectin-gold complexes for electron microscopic localization of glycoconjugates on thin sections J Histochem Cytochem 1983;31:987-99.

13 Taatles DJ, Schaub U, Roth J. Light microscopical detection of antigens and lectin binding sites with gold-labelled reagents on semi-thin Lowicryl K4M sections: usefulness of the photochemical silver reaction for signal amplification. Histochem $J$ 1987;19:235-45.

Requests for reprints to: Dr D R Davies, Department of Histopathology, St Thomas's Hospital, London SE1 7EH, England. 